\title{
Transcriptome analysis reveals ginsenosides biosynthetic genes, microRNAs and simple sequence repeats in Panax ginseng C. A. Meyer
}

Chunfang Li', Yingjie Zhu' ${ }^{1}$, Xu Guo ${ }^{1}$, Chao Sun ${ }^{1}$, Hongmei Luo ${ }^{1}$, Jingyuan Song ${ }^{1}$, Ying Li ${ }^{1}$, Lizhi Wang ${ }^{1}$, Jun Qian ${ }^{1}$ and Shilin Chen ${ }^{1,2^{*}}$

\begin{abstract}
Background: Panax ginseng C. A. Meyer is one of the most widely used medicinal plants. Complete genome information for this species remains unavailable due to its large genome size. At present, analysis of expressed sequence tags is still the most powerful tool for large-scale gene discovery. The global expressed sequence tags from $P$. ginseng tissues, especially those isolated from stems, leaves and flowers, are still limited, hindering in-depth study of $P$. ginseng.

Results: Two 454 pyrosequencing runs generated a total of 2,423,076 reads from $P$. ginseng roots, stems, leaves and flowers. The high-quality reads from each of the tissues were independently assembled into separate and shared contigs. In the separately assembled database, 45,849, 6,172, 4,041 and 3,273 unigenes were only found in the roots, stems, leaves and flowers database, respectively. In the jointly assembled database, 178,145 unigenes were observed, including 86,609 contigs and 91,536 singletons. Among the 178,145 unigenes, 105,522 were identified for the first time, of which $65.6 \%$ were identified in the stem, leaf or flower cDNA libraries of $P$. ginseng. After annotation, we discovered 223 unigenes involved in ginsenoside backbone biosynthesis. Additionally, a total of 326 potential cytochrome P450 and 129 potential UDP-glycosyltransferase sequences were predicted based on the annotation results, some of which may encode enzymes responsible for ginsenoside backbone modification. A BLAST search of the obtained high-quality reads identified 14 potential microRNAs in $P$. ginseng, which were estimated to target 100 protein-coding genes, including transcription factors, transporters and DNA binding proteins, among others. In addition, a total of 13,044 simple sequence repeats were identified from the 178,145 unigenes.

Conclusions: This study provides global expressed sequence tags for P. ginseng, which will contribute significantly to further genome-wide research and analyses in this species. The novel unigenes identified here enlarge the available $P$. ginseng gene pool and will facilitate gene discovery. In addition, the identification of microRNAs and the prediction of targets from this study will provide information on gene transcriptional regulation in $P$. ginseng. Finally, the analysis of simple sequence repeats will provide genetic makers for molecular breeding and genetic applications in this species.
\end{abstract}

Keywords: Expressed sequence tag, microRNA, Simple sequence repeats, Ginsenoside, Panax ginseng C. A. Meyer

\footnotetext{
* Correspondence: slchen@implad.ac.cn

${ }^{1}$ Chinese Academy of Medical Sciences \& Peking Union Medical College, Institute of Medicinal Plant Development, Beijing 100094, China

${ }^{2}$ China Academy of Chinese Medical Sciences, Institute of Chinese Materia Medica, Beijing 100700, China
}

\section{Biomed Central}

(c) 2013 Li et al.; licensee BioMed Central Ltd. This is an Open Access article distributed under the terms of the Creative Commons Attribution License (http://creativecommons.org/licenses/by/2.0), which permits unrestricted use, distribution, and reproduction in any medium, provided the original work is properly cited. 


\section{Background}

Panax ginseng C. A. Meyer is a widely used medicinal plant with multiple clinical and pharmacological effects related to cancer, diabetes and cardiovascular disease. It also promotes immune and central nervous system function as well as relieving stress [1]. The major bioactive components of $P$. ginseng are the ginsenosides, a group of dammarane- and oleanane-type triterpenoid saponins. The total ginsenoside content is highest in the flower, followed by the root, leaf and stem [2]. The large size and high complexity of the $P$. ginseng genome, which is reportedly tetraploid and $\sim 3.2 \mathrm{~Gb}$ in size [3], has made it difficult to obtain a complete genomic sequence for this species. Many researchers have obtained genomic information for $P$. ginseng by employing expressed sequence tags (ESTs), which are considered an efficient tool for gene discovery, especially in plants lacking an assembled genome [4]. Previous studies have generated ESTs derived from $P$. ginseng roots, rhizomes, seeds and leaves using the Sanger method [5-8]. However, as this method has a high cost and is very time consuming, only 17,773 ESTs obtained using this technique have been deposited in NCBI to date. Next-generation sequencing (NGS) technologies provide a rapid and economical way to sequence extremely large amounts of genetic material $[9,10]$. For example, Chen et al. generated 217,529 ESTs from 11-year-old $P$. ginseng roots via 454 sequencing [11]. Given that different tissues exhibit specific gene expression patterns, it is necessary to obtain the global transcriptome of other tissues to obtain full genomic information for P. ginseng.

ESTs are considered to represent a reliable source of data for predicting microRNAs (miRNAs) and their targets, especially in species without complete genome information [12]. miRNAs are important regulators in a wide range of developmental processes in plants, including cell proliferation, the stress response, metabolism, inflammation and signal transduction $[13,14]$. miRNAs have been identified successfully from plant EST databases based on sequence conservation and characteristic miRNA features [15-17]. However, miRNAs have not yet been identified from $P$. ginseng.

Simple sequence repeats (SSRs), also termed microsatellites, are nucleotide motifs consisting of tandem repeats of two to six base pairs. It is more likely for SSRs from ESTs (EST-SSRs) to be tightly linked to specific gene functions and perhaps even play a direct role in controlling important agronomic traits [18]. To date, only 251 SSRs have been identified in P. ginseng [19].

In the present study, we globally sequenced the transcriptomes of the roots, stems, leaves and flowers of 4-year-old P. ginseng plants. Novel and tissue-specific $P$. ginseng unigenes were obtained. Furthermore, our database includes all of the genes encoding enzymes involved in ginsenoside backbone biosynthesis and modification. Based on the obtained unigenes, we also identified 14 potential $P$. ginseng miRNAs and 100 of their potential target genes. Moreover, a total of 13,044 EST-SSRs were identified from the $P$. ginseng unigene dataset, which will facilitate marker-assisted breeding of P. ginseng.

\section{Results and discussion}

\section{Sequencing and de novo assembly}

To characterize the transcriptome of $P$. ginseng and generate expression profiles, we sequenced cDNA samples from four $P$. ginseng tissues (root, stem, leaf and flower) using a Roche/454 GS-FLX (Titanium) pyrosequencing machine. One half run was performed for each sample, yielding approximately 2.42 million raw reads, ultimately totaling $\sim 1.01$ billion base pairs (bp). Of these ESTs, $70.2 \%$ were over $400 \mathrm{bp}$ in length. The size distributions of the raw reads from the four samples are shown in Additional file 1 . To acquire high-quality reads, we filtered out adapter sequences and reads that were shorter than $50 \mathrm{bp}$. The high-quality reads from each sample were then used to build a de novo assembly using GS De NovoAssembler software, v2.6, both for each tissue individually and for all tissues as a group. The size distributions of the contigs from each tissue are presented in Additional file 2. After assembling the reads from all four tissues together, the generated contigs ranged from 100 to $7,858 \mathrm{bp}$, with an average size of $468.7 \mathrm{bp}$, while the size of the singletons ranged from 100 to $691 \mathrm{bp}$, averaging $382.9 \mathrm{bp}$. We obtained 178,145 unique sequences, totaling approximately $75.6 \mathrm{Mb}$. All of the high-quality reads generated in this study have been deposited at NCBI and can be accessed in the Sequence Read Achieve (SRA) Sequence Database under project accession number SRP015263. This Transcriptome Shotgun Assembly project has been deposited at DDBJ/EMBL/ GenBank under accession GAAG00000000. The version described in this paper is the first version, GAAG0100 0000. A summary of the 454 sequencing and assembly results for the four tissues is shown in Additional file 3, and the data for together assembly is presented in Table 1.

The current $P$. ginseng EST library found in the TSA database contains 15,357 ESTs from 11-year-old root tissue [11]. These ESTs are included in the 33,903 homologous unigenes revealed in the 4-year-old $P$. ginseng root transcriptome examined in the present study. Furthermore, 82,666 novel $P$. ginseng root unigenes were discovered in this study, some of which may be specifically expressed in 4-year-old roots. Whereas there are 32,441 ESTs in the NCBI database that were obtained via 454 and Sanger sequencing, 72,623 homologous genes and 105,522 novel unigenes were discovered in the present study. The large quantity of novel unique 
Table 1 Summary of the total 454 sequencing and the assembly results for $P$. ginseng four tissues

\begin{tabular}{lll}
\hline & $\begin{array}{l}\text { No. of } \\
\text { sequences }\end{array}$ & $\begin{array}{l}\text { No. of } \\
\text { bases }\end{array}$ \\
\hline High-quality reads & $2,423,076$ & $980,165,285$ \\
$\begin{array}{l}\text { Average high-quality read length (bp) } \\
\text { Reads used in assembly }\end{array}$ & 404.5 & \\
Number of contigs $\geq 100 \mathrm{bp}$ & $2,151,832$ & $863,992,539$ \\
Average length of contigs (bp) & 86,609 & $40,589,347$ \\
Range of contig lengths (bp) & 468.7 & \\
Number of singletons $\geq 100$ bp & $100-7,858$ & \\
$\begin{array}{l}\text { Average length of singletons (bp) } \\
\text { Range of singleton lengths (bp) }\end{array}$ & 91,536 & $35,032,649$ \\
$\begin{array}{l}\text { Number of unigenes (contigs and } \\
\text { singletons) }\end{array}$ & $100-691$ & \\
Total coverage (bp) & 178,145 & \\
\hline
\end{tabular}

sequences identified in this study constitutes a powerful resource for $P$. ginseng researchers.

\section{Functional annotation and candidate genes encoding} enzymes involved in the biosynthesis of ginsenosides To obtain the most informative and complete annotation, all of the contigs from roots, stems, leaves and flowers were annotated separately. The numbers and percentages of the annotated unique sequences are presented in Additional file 4. In total, 94,535 unique sequences presented at least one significant match in the public databases. The remaining unigenes that were not annotated appeared to be either $P$. ginseng-specific genes or homologous genes with unknown functions in other species.
Based on the annotation results, transcripts encoding all of the known enzymes involved in ginsenoside biosynthesis and modification were identified in our dataset (Figure 1). In most cases, multiple unigenes were annotated as corresponding to the same enzyme (Additional file 5). Such unigenes may represent different fragments of a single transcript or different members of a gene family.

Chen et al. [11] analyzed the transcriptome of 11-yearold $P$. ginseng roots and discovered many genes involved in ginsenoside biosynthesis. However, several genes encoding key enzymes involved in ginsenoside skeleton biosynthesis were absent, such as mevalonate kinase $(M V K)$, geranylgeranyl pyrophosphate synthase (GPS), amyrin synthase $(A S)$ and dammarendiol synthase (DS). From the global tissue transcriptome analysis, we obtained higher coverage genetic information and more candidate genes for further analysis. MVK, GPS and DS were found in our 4-year-old root cDNA library (Table 2). This difference of results may be due to these three enzymes being actively expressed in the 4-yearold $P$. ginseng root, but expressed only at a low level in the 11-year-old $P$. ginseng root, or to the high coverage of the 4-year-old root transcriptome. $A S$ was absent in both the 4- and 11-year-old $P$. ginseng roots but was found in the 4-year-old $P$. ginseng stems, leaves and flowers in the transcriptome database. This result may indicate that the biosynthsis of oleanane-type ginsenosides might be actived in the stem, leaf and flower of 4-year-old P. ginseng, but not in the root of the 4- and 11-year-old P. ginseng.

Specific CYP450s catalyze the conversion of damma renediol-II or $\beta$-amyrin to various ginsenosides. Han et al. identified the involvement of CYP716A47 in the hydroxylation of the $\mathrm{C}-12$ position to yield protopanaxadiol

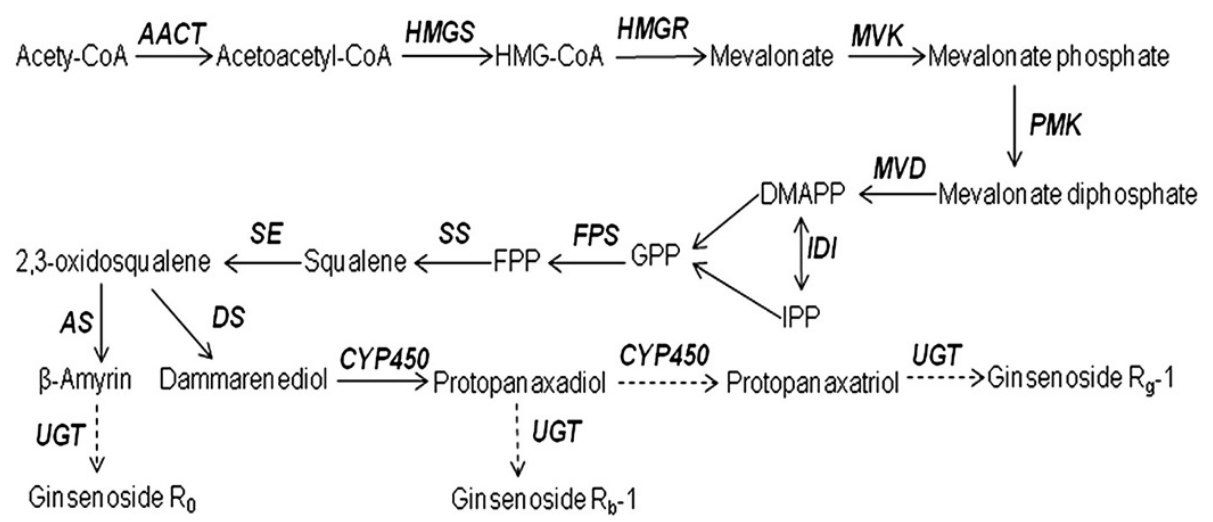

Figure 1 Putative ginsenoside biosynthesis pathway. Candidate genes identified in this study are shown in bold. Acetyl-CoA acetyltransferase (AACT), HMG-CoA synthase (HMGS), HMG-CoA reductase (HMGR), mevalonate kinase (MVK), phosphomevalonate kinase (PMK), mevalonate diphosphate decarboxylase (MVD), isopentenyl diphosphate isomerase (IDI), geranylgeranyl pyrophosphate synthase (GPS), farnesyl diphosphate synthase (FPS), squalene synthase (SS), squalene epoxidase (SE), amyrin synthase (AS), dammarendiol synthase (DS), UDP glycosyltransferase (UGT) and cytochrome P450 (CYP450). 
Table 2 Comparison of the unigene numbers from tissues of 4- and 11-year-old P. ginseng

\begin{tabular}{|c|c|c|c|c|}
\hline \multirow{2}{*}{$\begin{array}{l}\text { Gene } \\
\text { name }\end{array}$} & \multirow{2}{*}{$\begin{array}{l}\text { EC } \\
\text { number }\end{array}$} & \multicolumn{3}{|c|}{ Unigene number } \\
\hline & & $\begin{array}{c}\text { Four tissues of 4-year } \\
\text { old plant }\end{array}$ & $\begin{array}{c}\text { 4-year-old } \\
\text { root }\end{array}$ & $\begin{array}{l}\text { 11-year- } \\
\text { old root }\end{array}$ \\
\hline AACT & 2.3.1.9 & 18 & 5 & 4 \\
\hline HMGS & 2.3.1.10 & 8 & 1 & 2 \\
\hline$H M G R$ & 1.1 .1 .34 & 32 & 5 & 2 \\
\hline MVK & 2.7.1.36 & 9 & 3 & 0 \\
\hline PMK & 2.7 .4 .2 & 16 & 8 & 2 \\
\hline MVD & 4.1.1.33 & 11 & 3 & 1 \\
\hline$|D|$ & 5.3.3.2 & 15 & 1 & 2 \\
\hline GPS & 2.5.1.29 & 45 & 2 & 0 \\
\hline FPS & $\begin{array}{l}2.5 .1 .1 \\
2.5 .1 .10\end{array}$ & 14 & 2 & 3 \\
\hline SS & 2.5.1.21 & 22 & 1 & 1 \\
\hline SE & 1.14.99.7 & 21 & 3 & 1 \\
\hline AS & & 6 & 0 & 0 \\
\hline DS & & 16 & 1 & 0 \\
\hline
\end{tabular}

[20]. In this study, 326 putative CYP450 unigenes were identified, including the CYP716A47 gene. Based on our CYP450 pool, further research will be performed to identify other CYP450s that may participate in ginsenoside biosynthesis in $P$. ginseng. Glycosylation, the transfer of activated saccharides to an aglycone substrate, is the predominant type of modification that occurs in the last step of ginsenoside biosynthesis. Glycosylation of dammaraneand oleanane-type aglycones is required for ginsenoside bioactivity. In this study, 129 putative UDP glycosyltransferase (UGT) unigenes were found in the $P$. ginseng transcriptome, of which 6 showed a high sequence similarity to the candidate glucosidase genes identified in $P$. quinquefolius by Sun et al. [21]. These putative $P$. ginseng UGTs included contig89150, contig89599, contig48582, contig18298, contig76094 and contig72547 from the database derived from assembling all tissues simultaneously. The roles of these candidate UGT unigenes in ginsenoside biosynthesis need to be further characterized.

\section{Comparative and Gene Ontology analyses of $P$. ginseng root, stem, leaf and flower unigenes}

Unigene sequences of the $P$. ginseng four tissues were compared with each other and was shown by a Venn diagram (Figure 2). The four tissues shared 50,957 unigenes, which likely include housekeeping genes playing key roles in $P$. ginseng. The number of unigenes only can be found in the database of each tissue was 45,849 for the root, 6,172 for the stem, 4,041 for the leaf and 3,273 for the flower. The number of unigenes which can only be found in root database was highest among the four tissues, maybe because that the root expressed

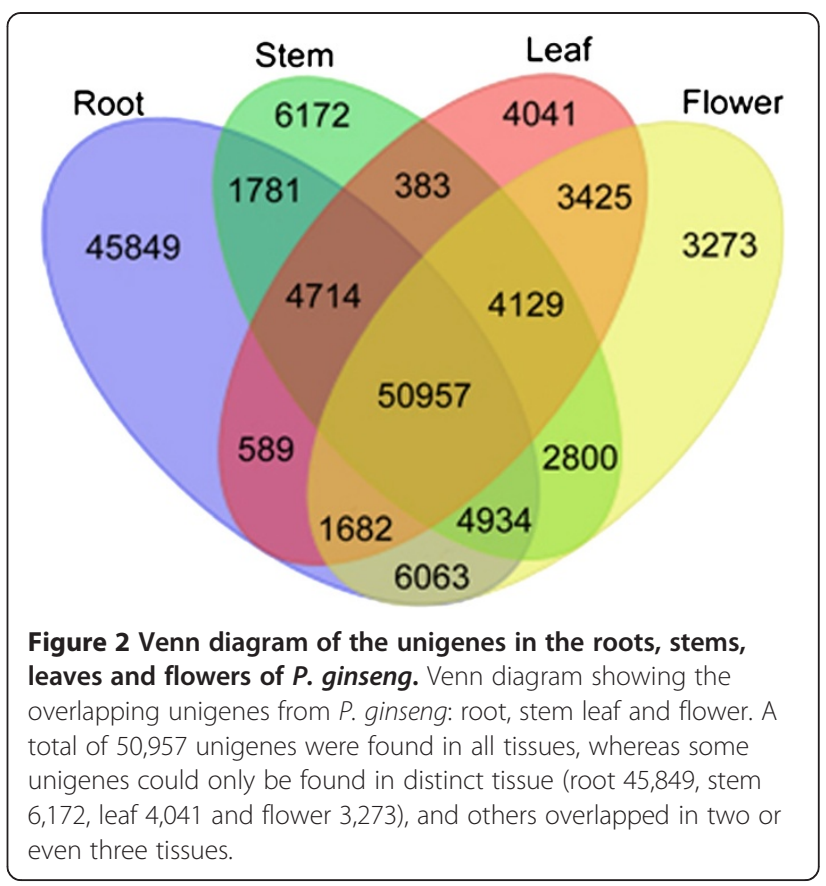

more genes than the other three tissues. The unigenes only found in the stem, leaf and flower databases corresponded to $65.6 \%$ of the novel genes discovered in our study and might represent genes controlling development in different tissues.

Gene Ontology (GO) is widely used to standardize the representation of genes across species and to provide a controlled vocabulary of terms for describing gene products [22]. The contigs from four tissues were assigned GO terms based on BlastP searches against sequences with products whose functions were previously identified. These GO terms were summarized into three main GO categories (biological process, cellular component and molecular function) according to the standard GO terms and 23 sub-categories (Figure 3). In each tissue, the biological process category comprised the majority of GO annotations, followed by the cellular component and molecular function categories. The percentage of each sub-category in each tissue was quite different. Notably, within the biological process category in the root transcriptome, multicellular organismal process and growth were the most dominant subcategories, reflecting the rapid growth occurring in the root. In the other three tissues, the dominant subcategory was response to stimulus. For the cellular component category, the most highly represented subcategory in roots and leaves was extracellular region part, while in stems, it was organelle and in flowers, it was extracellular region. Under the molecular function category, the main subcategory in roots and leaves was catalytic activity, while it was transcription regulator activity in stems and binding in flowers. These GO annotations represent a general 


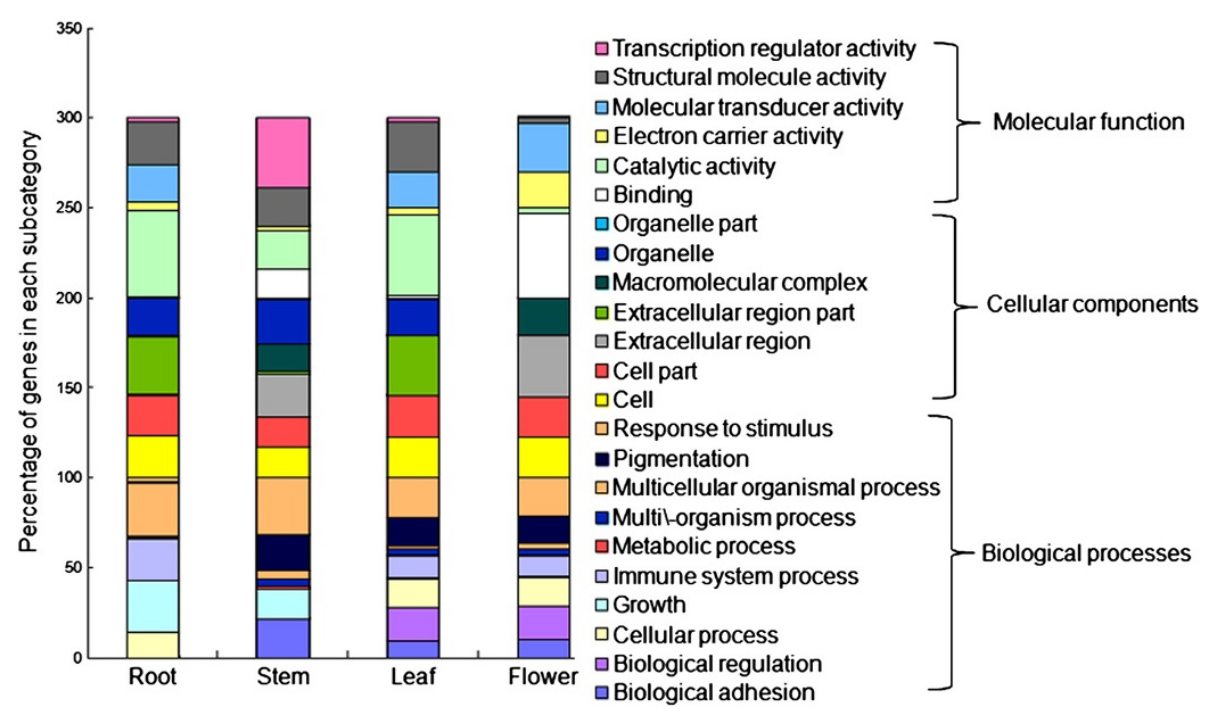

Figure 3 Functional classification of unigenes in the four $P$. ginseng tissues based on Gene Ontology categories. Unique sequences were classified into 23 gene ontology categories under three major categories: cellular components, molecular functions and biological processes.

gene expression profile signature of the different tissues of $P$. ginseng and demonstrate that the genes expressed in these different tissues encode diverse structural, regulatory and stress response proteins.

\section{Analysis of the predominant transcripts in $P$. ginseng roots, stems, leaves and flowers}

The abundance of particular transcripts within a specific tissue provides clues about the biological processes occurring there. The most highly expressed genes observed in each cDNA library are listed in Table 3 . The genes encoding catalase and superoxide dismutase were present in all four cDNA libraries and presented particularly high levels in the root. Catalase and superoxide dismutase are two antioxidant enzymes that play key roles in antioxidant defense systems and in the protection of plant cells against oxidative damage caused by reactive oxygen species [23]. Other abundant transcripts in the root-derived library encoded a latex-like protein, ribonuclease-like storage protein, 1,4-alpha-glucan branching enzyme and some proteins with unknown functions, which could be $P$. ginseng-specific proteins.

In the stem-derived cDNA library, the most highly expressed gene encoded phloem protein 2 (PP2)-like protein. PP2 is involved in the assimilate stream and has the capacity to interact with the mesophyll plasmodesmata to increase their size exclusion limit as well as cell-to-cell trafficking [24]. The second most highly expressed gene encodes a dehydration-related protein involved in the response to environmental stresses [25]. Genes encoding photosynthesis-related proteins, such as chlorophyll a-b binding proteins, peroxidase and the photosystem II PsbK protein, were also found to be abundant in the stem. Genes encoding chlorophyll a-b binding proteins were the most highly expressed genes in the stem-, leaf- and flower-derived libraries, and the root-derived cDNA libraries also contained several transcripts of chlorophyll $a-b$ binding genes. This difference may be because stems, leaves and flowers are all chloroplast-containing tissues, whereas there are no chloroplasts in the tissues found in the root.

The most highly expressed genes in the leaf-derived cDNA library encoded proteins including ribulose bisphosphate carboxylase, photosystem II $10 \mathrm{kDa}$ polypeptide and the chlorophyll a-b binding protein, as well as some proteins with an undefined function. Ribulose biphosphate carboxylase was also the most abundant transcript in the flower-derived libraries. Ribulose bisphosphate carboxylase catalyzes the initial step in the photosynthetic fixation of carbon dioxide [26].

Histone H4, aquaporin and a photosystem II $10 \mathrm{kDa}$ polypeptide represented the three dominant transcripts in the flower-derived cDNA library. Histone $\mathrm{H} 4$ acetylation within euchromatic and heterochromatic domains plays a key role in DNA replication [27]. In higher plants, aquaporins are water channel proteins that facilitate the passage of water through biological membranes and play a crucial role in plant growth [28].

All of the predominantly expressed transcripts in each of these four tissue-derived cDNA libraries belong to the group of 50,957 genes shared by the four tissues. These genes are normally associated with housekeeping functions and play key roles in P. ginseng growth and development. Some housekeeping genes exhibit high expression levels in specific tissues, as observed for transport-related genes in the stem and photosynthesis-related genes in the 
Table 3 Predominant transcripts in the CDNA libraries generated from P. ginseng roots, stems, leaves and flowers

\begin{tabular}{|c|c|c|c|}
\hline Unigene ID & Subject & Annotation & No. of reads \\
\hline \multicolumn{4}{|l|}{ Root } \\
\hline contig03234 & vvi:100267971 & Hypothetical protein & 946 \\
\hline contig03264 & vvi:100267971 & Hypothetical protein & 921 \\
\hline contig08673 & sp|O24339| & Catalase & 904 \\
\hline contig 08301 & sp|P24669 & Superoxide dismutase & 872 \\
\hline contig01891 & gb|ACH72969.1| & Latex-like protein & 862 \\
\hline contig02117 & sp|P83618| & Ribonuclease-like storage protein & 846 \\
\hline contig08057 & ref|XP_002467304.1| & Hypothetical protein & 838 \\
\hline contig08066 & ref|XP_002488947.1| & Hypothetical protein & 788 \\
\hline contig08059 & pop:POPTR_265927 & Hypothetical protein & 780 \\
\hline contig09140 & vvi:100257371 & 1,4-alpha-glucan branching enzyme & 776 \\
\hline \multicolumn{4}{|l|}{ Stem } \\
\hline contig01514 & sp|Q9C8U9| & Phloem protein 2-like protein & 993 \\
\hline contig01413 & gb|ABF48477.1| & Panax ginseng dehydrin 4 & 953 \\
\hline contig04579 & pop:POPTR_820375 & Hypothetical protein & 948 \\
\hline contig02872 & sp|P04778| & Chlorophyll a-b binding protein 1 & 941 \\
\hline contig04578 & emb|CAH59408.1| & Hypothetical protein & 920 \\
\hline contig02875 & sp|P27495| & Chlorophyll a-b binding protein 40 & 915 \\
\hline contig04448 & sp|Q42580| & Peroxidase 21 & 911 \\
\hline contig02517 & pop:POPTR_718004 & Hypothetical protein & 890 \\
\hline contig02408 & ref|XP_002299525.1| & Hypothetical protein & 887 \\
\hline contig02549 & ref|YP_003934332.1| & Photosystem II PsbK protein & 859 \\
\hline \multicolumn{4}{|l|}{ Leaf } \\
\hline contig00303 & rcu:RCOM_0030020 & Ribulose bisphosphate carboxylase small chain & 19345 \\
\hline contig00304 & sp|P10795| & Ribulose bisphosphate carboxylase small chain $1 \mathrm{~A}$ & 18860 \\
\hline contig00004 & rcu:RCOM_1571640 & Ribulose bisphosphate carboxylase small chain 1B & 11627 \\
\hline contig00270 & rcu:RCOM_1158790 & Ribulose bisphosphate carboxylase small chain & 7879 \\
\hline contig00520 & rcu:RCOM_1158790 & Ribulose bisphosphate carboxylase small chain & 6692 \\
\hline contig00325 & sp|P23540| & Ribonuclease & 3909 \\
\hline contig01392 & sp|P49108| & Photosystem I| $10 \mathrm{kDa}$ polypeptide & 3202 \\
\hline contig00936 & sp|P27497| & Chlorophyll a-b binding protein M9 & 2041 \\
\hline contig00949 & sp|P13851| & Chlorophyll a-b binding protein 1 & 1955 \\
\hline contig02266 & ref|XP_002273037.1| & Hypothetical protein & 1785 \\
\hline \multicolumn{4}{|l|}{ Flower } \\
\hline contig01060 & rcu:RCOM_1158790 & Ribulose bisphosphate carboxylase small chain & 999 \\
\hline contig04296 & sp|P92919| & Chlorophyll a-b binding protein & 942 \\
\hline contig01114 & sp|Q40250| & Ribulose bisphosphate carboxylase small chain & 932 \\
\hline contig26298 & zma:100192931 & Histone H4 & 919 \\
\hline contig04288 & sp|P07371| & Chlorophyll $a-b$ binding protein AB80 & 887 \\
\hline contig05604 & ref|XP_002312129.1| & Hypothetical protein & 881 \\
\hline contig33033 & pop:POPTR_826664 & Hypothetical protein & 830 \\
\hline contig00507 & gb|AAX40470.1| & Hypothetical protein & 788 \\
\hline contig04848 & sp|Q40519| & Photosystem II 10 kDa polypeptide & 772 \\
\hline contig14522 & sp|P50156| & Probable aquaporin TIP1-1 & 754 \\
\hline
\end{tabular}


leaf. This phenomenon can be explained by the fact that the proteins encoded by these genes are responsible for the characteristic functions of the corresponding tissues.

\section{Identification and characterization of potential miRNAs in P. ginseng}

miRNAs are a class of noncoding, small endogenous RNAs, 22 nucleotides (nt) in length that have been shown to regulate gene expression at the post-transcrip tional level by targeting mRNAs for degradation or inhibiting protein translation [29]. There are currently 4,743 miRNAs that have been identified from 51 plant species deposited in the miRBase database [30]. However, no miRNAs have been identified in $P$. ginseng until now. Because only mature miRNA sequences (rather than precursor sequences) are conserved among plant species, mature miRNA sequences were used as queries for BLAST searches against the high-quality $P$. ginseng reads derived from our experiments. This process yielded 8,375 reads that were found to significantly match at least one mature miRNA sequence with no more than two mismatches and that could be related to either a target or an miRNA precursor sequence. A total of 3,707 noncoding reads were obtained after removing repeat and protein-coding sequences. Ultimately, we identified 14 candidate $P$. ginseng miRNAs with a proper miRNA precursor secondary structure and a minimal folding free energy index (MFEI) of at least 0.85 (Table 4).

Mature miRNA sequences can be located on either arm of the secondary stem-loop hairpin structure of the potential miRNA precursor (pre-miRNA). Of the 14 identified $P$. ginseng miRNAs, 4 were found to be located on the $5^{\prime}$ arm of the stem-loop hairpin structure, while 10 resided on the $3^{\prime} \mathrm{arm}$. The length of the putative $P$. ginseng miRNAs varied from 20 to $22 \mathrm{nt}$, with an average of $21 \pm 0.5 \mathrm{nt}$. The majority (10 out of 14 , or $71.4 \%$ ) of the miRNAs were $21 \mathrm{nt}$ in length. The length of the $P$. ginseng pre-miRNAs varied from 68 to $294 \mathrm{nt}$, averaging $143 \pm 67 \mathrm{nt}$. The length distribution of the miRNAs and their precursor sequences was similar to the distributions described in previous reports in other plant species $[12,31]$.

The minimal folding free energy (MFE) is important for the formation of RNA secondary structures. Generally, the lower the MFE, the more stable the secondary structure of a given RNA sequences. The average MFE value obtained in the present study for the $P$. ginseng miRNAs was $-54.78 \pm 21.82 \mathrm{kcal} / \mathrm{mol}$, with a range of -21.9 to $-99.2 \mathrm{kcal} / \mathrm{mol}$. The minimal folding free energy index (MFEI) is a criterion for distinguishing miRNAs from other RNAs. Previous studies have shown that a sequence is more likely to be a potential miRNA if it presents an MFEI value greater than 0.85 [32]. For the 14 newly identified $P$. ginseng miRNAs, the average MFEI was $1.04 \pm 0.17$, with a range of 0.85 to 1.55 . The secondary structures of the putative $P$. ginseng miRNA precursors are reported in Additional file 6.

\section{Target prediction for the $P$. ginseng miRNAs}

Identification of miRNA target genes, in addition to constituting indirect existence evidence of miRNAs, is a fundamental step for the determination of biological function for miRNAs. Evolutionarily conserved targets have been shown to be useful in testing the effectiveness

Table 4 Candidate miRNAs in $P$. ginseng

\begin{tabular}{|c|c|c|c|c|c|c|c|c|c|c|}
\hline miRNA & Query miRNAs & ML & Mature sequence & NM & Arm & GC\% & PL & MFE & MFEI & EST ID \\
\hline pgi-miR1128 & ssp-miR1128 & 21 & UGCUACUCCCUCCGUCCCAAA & 5 & $5^{\prime}$ & 22 & 290 & 99.2 & 1.55 & G8WERZI02JSLMP \\
\hline pgi-miR827 & tcc-miR827 & 21 & UUAGAUGAUCAUCAGCAAACA & 1 & $3^{\prime}$ & 32 & 117 & 43.2 & 1.15 & G8WERZI01D2S8J \\
\hline pgi-miR1439 & osa-miR1439 & 21 & UUUUGGAACGGAGGGAGUAUU & 4 & $3^{\prime}$ & 28 & 294 & 92.6 & 1.12 & G8WERZI01A17SA \\
\hline pgi-miR5658 & ath-miR5658 & 21 & 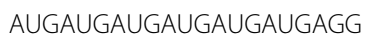 & 3 & $3^{\prime}$ & 39 & 80 & 34.7 & 1.11 & G9IMXQ301ESZFB \\
\hline pgi-miR396i-3p & gma-miR396i-3p & 21 & GUUCAAUAAAGCUGUGGGAAG & 1 & $3^{\prime}$ & 43 & 119 & 54.6 & 1.07 & G9IMXQ302GOGE4 \\
\hline pgi-miR390b & bdi-miR390b & 21 & AAGCUCAGGAGGGAUAGCGCC & 1 & $5^{\prime}$ & 39 & 175 & 72.5 & 1.06 & G9IMXQ302H2ZM1 \\
\hline pgi-miR5021 & ath-miR5021 & 20 & GAAGAAGAAGAAGAAGAAAA & 3 & $5^{\prime}$ & 32 & 134 & 44.8 & 1.04 & G9IMXQ301DYCTK \\
\hline pgi-miR156b & vun-miR156b & 21 & UGACAGAAGACUAGAGAGCAC & 1 & $5^{\prime}$ & 44 & 112 & 49.6 & 1.01 & G9IMXQ302IGRXR \\
\hline pgi-miR403b & tcc-miR403b & 21 & UUAGAUUCACGCACAAACUCG & 3 & $3^{\prime}$ & 41 & 117 & 46.8 & 0.98 & G9IMXQ301CU7GZ \\
\hline pgi-miR172 & aau-miR172 & 22 & UGAGAAUCUUGAUGAUGCUGCA & 3 & $3^{\prime}$ & 44 & 171 & 71 & 0.94 & G9IMXQ301B4R7M \\
\hline pgi-miR408 & vun-miR408 & 21 & AUGCACUGCCUCUUCCCUGGC & 4 & $3^{\prime}$ & 50 & 115 & 53.6 & 0.93 & G9IMXQ301BH247 \\
\hline pgi-miR399d & tcc-miR399d & 21 & UGCCAAAGGAGAUUUGCCCGG & 3 & $3^{\prime}$ & 41 & 122 & 45.2 & 0.9 & G8WERZI01A00R5 \\
\hline pgi-miR482 & gra-miR482 & 22 & UCUUGCCAAUUCCUCCCAUUCC & 3 & $3^{\prime}$ & 43 & 97 & 37.2 & 0.89 & G9IMXQ301C4CDG \\
\hline pgi-miR3441.1 & aly-miR3441.1 & 20 & UUCAAAGCCUCUUUGAAGGA & 5 & $3^{\prime}$ & 38 & 68 & 21.9 & 0.85 & G9IMXQ301DXOD1 \\
\hline
\end{tabular}

$M L$, mature length; $N M$, number of mismatches in miRNA/miRNA* duplexes; Arm, location of mature miRNA; $P L$, precursor length; $M F E$, minimal folding free energy; MFEI, minimal folding free energy index; EST ID, the high quality reads number which can be accessed in the SRA Sequence Database under project accession number SRP015263. 
of miRNA target detection. A perfect, or near perfect, complementarity between an miRNA and its target mRNA, which is a peculiar feature of plant miRNAs, provides a powerful tool for the identification of target genes through BLAST analysis of mature miRNA sequences against EST sequences. After carefully considering the alignment results, we predicted at least one target for 7 miRNAs and a total of 100 potential targets for 14 miRNAs (Additional file 7). There were 37 and 51 targets predicted for miR5658 and miR5021, respectively, while the targets associated with other miRNAs were much less abundant, or may have failed to be sequenced. Zhou et al. detected a large number of targets for miR156 and miR396 and a small number for miR162, miR167, miR395, miR398 and miR399 in rice [33]. miRNAs with a large number of targets may represent nodes in gene regulatory networks, while those with a small number of targets may act through more specialized pathways.

Several studies have demonstrated that miRNAs can target transcription factors that control plant growth and development $[13,31]$. In the present study, the putative $P$. ginseng miR172 was predicted to target the transcription factor APETALA2 (AP2), which plays an important role in the control of the flowering time and floral morphology. miR172 has also been shown to target AP2 in tobacco, the opium poppy and Brassica oleracea [34-36]. Aukerman and Sakai found that overexpression of miR172, which targets AP2, causes early flowering and suppresses the floral organ specification in Arabidopsis [37]. In addition, this study suggested that MYB proteins might be the target of miR5021 in P. ginseng. Previous studies have also shown that MYB proteins may be targeted by miR5021 in B. oleracea [36]. The MYB transcription factors found in plants constitute a superfamily of proteins with a conserved MYB DNA binding domain that play a regulatory role in developmental processes and defense responses [38]. Zinc finger protein family members were predicted to be targeted by miR1128, miR5658 and miR5021. Zinc-finger proteins orchestrate the responses of plants to changes in environmental conditions and play a central role in reactive oxygen and abiotic stress signaling in Arabidopsis [39]. Other miRNAs identified in this study, such as miR403b, miR172, miR3441.1 and miR1439, can be considered putative regulators of gene expression at the protein level.

\section{Identification of simple sequence repeats (SSRs) in P. ginseng}

SSRs are one of the most powerful types of molecular marker because of their relative abundance and ease of generation, and they have been widely applied for molecular-assisted selection (MAS) in plant breeding programs [40]. SSR markers derived from expressed sequence tags are likely to be even more transferable across lines, populations and species than random genomic SSRs [41]. In this study, a total of 13,044 SSRs were identified from 178,145 unigenes, with 1,582 of the $P$. ginseng unigenes containing at least two SSRs. The observed frequency of unigenes was $7.3 \%$, and the distribution density was 172.5 per Mb. As is shown in Table 5, the most abundant repeat type was dinucleotides $(51.0 \%$, $6,659)$, and the most common number of tandem repeats was $6(24.4 \%, 3,179)$. The dominant repeat motif was AG/CT, with a frequency of $24.5 \%$ (Additional file 8 ). The primer pairs for each SSR conforming to a series of primer-designing parameters (see Methods) are offered in Additional file 9 for further investigation of the potential of these SSRs as genetic markers.

\section{Conclusions}

In this study, a large-scale EST investigation was performed in root, stem, leaf and flower tissues from $P$. ginseng. The obtained EST dataset provides a comprehensive resource for gene discovery and genetic analyses in P. ginseng. The genes identified in this study will help to decipher the molecular mechanisms of secondary metabolism in P. ginseng. Moreover, this study identified putative miRNAs from $P$. ginseng and their targets, thus representing a foundation for further research into transcriptional regulation. Finally, the large set of SSRs identified in this work

Table 5 Frequencies of repeat types with repeat numbers in $P$. ginseng EST-SSRs

\begin{tabular}{|c|c|c|c|c|c|c|c|c|c|c|}
\hline \multirow[t]{2}{*}{ Motif length } & \multicolumn{8}{|c|}{ Repeat number } & \multirow[t]{2}{*}{ Total } & \multirow[t]{2}{*}{$\%$} \\
\hline & 4 & 5 & 6 & 7 & 8 & 9 & 10 & $>10$ & & \\
\hline Di & - & - & 2,169 & 1,530 & 999 & 631 & 414 & 916 & 6,659 & 51.0 \\
\hline Tri & - & 1,868 & 786 & 408 & 226 & 118 & 67 & 162 & 3,635 & 27.9 \\
\hline Tetra & 990 & 255 & 116 & 57 & 22 & 7 & 1 & 7 & 1,455 & 11.2 \\
\hline Penta & 358 & 116 & 40 & 15 & 1 & 1 & 1 & 0 & 532 & 4.1 \\
\hline Hexa & 442 & 187 & 68 & 34 & 15 & 5 & 6 & 6 & 763 & 5.8 \\
\hline total & 1,790 & 2,426 & 3,179 & 2,044 & 1,263 & 762 & 489 & 1,091 & 13,044 & - \\
\hline$\%$ & 13.7 & 18.6 & 24.4 & 15.7 & 9.7 & 5.8 & 3.7 & 8.4 & - & - \\
\hline
\end{tabular}


provides abundant genetic markers for molecular breeding and genetic applications in this species.

\section{Methods}

\section{Plant material}

Actively growing 4-year-old ginseng ( $P$. ginseng C. A. Meyer) was harvested from a field plot in Kuandian County, Liaoning Province, China, on June 27, 2011. At that time, the temperature ranged from $14.3 \sim 24.7^{\circ} \mathrm{C}$, averaging $19.4^{\circ} \mathrm{C}$. The four seasons in Kuandian County are distinct. A majority of the annual rainfall occurs in July and August. The monthly 24-hour average temperatures range from $-11.5^{\circ} \mathrm{C}$ in January to $22.5^{\circ} \mathrm{C}$ in July, while the annual mean is $6.5^{\circ} \mathrm{C}$. The average relative humidity is $70 \%$, and the frost-free period is 140 days. Main roots, stems, leaves and flower buds were collected separately from a single plant and cut into small pieces followed immediately by storage in liquid nitrogen until further processing.

\section{RNA preparation}

Total RNA was isolated from roots, stems, leaves and flowers using the RNeasy Plus Mini kit (Qiagen, Valencia, CA, USA). Quality control was performed in the samples using RNA 6000 Nano LabChips with Bioanalyzer 2100 (Agilent Technologies, PaloAlto, CA, USA), and the obtained concentrations were assessed using a NanoDrop ND-1000 spectrophotometer (Nano-Drop Technologies, Wilmington, DE, USA) before processing. The RNA samples were treated with TURBO DNase (Ambion, Austin, TX, USA) at a concentration of 1.5 units/ $\mu \mathrm{g}$ of total RNA prior to cDNA synthesis.

\section{cDNA synthesis and sequencing}

Four cDNA libraries were constructed from the roots, stems, leaves and flowers of $P$. ginseng. First-strand cDNA was produced from $2 \mu \mathrm{g}$ of total RNA extracted from each of the $P$. ginseng tissues using the SMART cDNA synthesis kit (Clontech, Palo Alto, CA, USA) according to the manufacturer's instructions, with slight modifications, as described in a previous report [21]. For double-stranded cDNA (ds cDNA) synthesis, the cDNA was amplified using PCR Advantage II polymerase (Clontech, Palo Alto, CA, USA) with the following thermal profile: $1 \mathrm{~min}$ at $95^{\circ} \mathrm{C}$, followed by 19 cycles of $95^{\circ} \mathrm{C}$ for $15 \mathrm{~s}, 65^{\circ} \mathrm{C}$ for $30 \mathrm{~s}$ and $68^{\circ} \mathrm{C}$ for $6 \mathrm{~min}$. Then, $5 \mu \mathrm{l}$ of the obtained PCR products were electrophoresed in a $1 \%$ agarose gel to determine the amplification efficiency. Finally, all of samples were amplified over 12 cycles. Approximately $13 \mu \mathrm{g}$ of the amplified ds cDNA was purified using the Pure-Link ${ }^{\mathrm{TM}}$ PCR purification kit (Invitrogen Life Technologies Corp, Carlsberg, Calif, USA) and the cDNA was subsequently treated with BsgI (NEB, Ipswich, MA, USA) overnight and recovered using the QIAquick PCR Purification Kit (Qiagen, Valencia, CA, USA).

Next, 500 ng of ds cDNA from each tissue was used for shotgun cDNA library construction according to the manual of the GS FLX Titanium Rapid Library Preparation Kit (454 Life Sciences Corp, Branford, CT, USA). The DNA was nebulized for 1 minute and then end-repaired using T4 DNA polymerase and T4 polynucleotide kinase. Adaptors were blunt-end ligated to the fragment ends using T4 DNA ligase. AMPure beads (Agencourt Bioscience Corp, Beverly, MA, USA) were employed to remove small DNA fragments and to collect DNA fragments between $600 \mathrm{bp}$ and $900 \mathrm{bp}$ in length.

Using emulsion PCR, the DNA molecules in the shotgun library were amplified with the GS FLX Titanium LV emPCR package (454 Life Sciences Corp, Branford, CT, USA), according to the manufacturer's recommendations. After amplification, the beads bound to amplified molecules were collected, and the emulsion oil was removed via washing, according to the manufacturer's protocol. Beads bound to a sufficient number of copies of the clonally amplified library fragments were selected using a specified enrichment procedure and were subsequently counted with a Multisizer 3 Coulter Counter (Beckman Coulter, Fullerton, CA, USA) prior to sequencing.

Following emulsion PCR enrichment, the selected beads were loaded into the wells of a Titanium Series PicoTiterPlate device via centrifugation. Then, 454 sequencing was performed according to the manufacturer's instruction manual (454 Life Sciences Corp, Branford, CT, USA). Image analysis, signal processing, and base calling were conducted using Newbler 2.3 software (454 Life Sciences Corp, Branford, CT, USA).

\section{EST assembly and data analysis}

The raw 454 reads were screened and trimmed for weak signals using GS FLX pyrosequencing software to yield high-quality reads. The primer and adapter sequences were trimmed from the high-quality sequences to obtain clean ESTs. Then, sequences shorter than $50 \mathrm{bp}$ were removed. Finally, the data from the 454 reads was assembled into unigenes (containing contigs and singletons) using GS De NovoAssembler software, v2.6 (454 Life Sciences Corp, Branford, CT, USA). Functional annotation was carried out against a series of nucleotide and protein databases, including the Swiss-Prot [42], InterPro [43], Kegg [44], Nr [45] and Nt [46] databases, using BLAST (version 2.2.17) with a common significance threshold cutoff of $E$-value $\leq 1 \mathrm{e}-5$. The functional categories of these unique sequences were further analyzed according to $\mathrm{GO}$ terms based on InterPro GO slim provided by InterPro [43]. 


\section{Homology-based searches for miRNAs and target prediction}

A set of known miRNAs was downloaded from miRBase (version 18.0, November 2011), comprising a total of 4,743 mature miRNA sequences from 51 plant species [30]. A homology-based search for miRNAs in $P$. ginseng was carried out using previously reported methods [12,31]. Subsequently, miRNA targets were predicted according to the method described in a previous report [31].

\section{SSRs detection and primer design}

To detect SSRs in P. ginseng, we performed SSR analysis of the obtained unigenes using the microsatellite identification tool MISA [47]. The parameters were designed for identifying di-, tri-, tetra-, penta- and hexa-nucleotide motifs with a minimum of $6,5,4,4$ and 4 repeats, respectively [48]. Primer3 software was employed to design flanking primers for detecting microsatellites [49]. The main primer design parameters were set as follows: PCR products ranging from 100 to $300 \mathrm{nt}$, primer lengths ranging from 18 to $24 \mathrm{nt}$ (with an optimum of $20 \mathrm{nt}$ ), a $60^{\circ} \mathrm{C}$ optimal annealing temperature, and a GC content from $40 \%$ to $65 \%$ (with an optimum of 50\%) [48].

\section{Additional files}

Additional file 1: Length distributions of reads. TIFF document for the length distributions of $P$. ginseng four tissues reads.

Additional file 2: Length distributions of contigs. TIFF document for the length distributions of $P$. ginseng four tissues contigs.

Additional file 3: Summary of the $\mathbf{4 5 4}$ sequencing and assembly for $P$. ginseng four tissues. DOCX document for the summary of 454 sequencing and assembly data.

Additional file 4: Summary of annotation statistics against public databases for $P$. ginseng four tissues. DOCX document for the summary of annotation results.

Additional file 5: Transcripts involved in ginsenoside skeleton biosynthesis in $P$. ginseng four tissues. DOCX document for the number of ginsenoside skeleton biosynthesis genes in $P$. ginseng each tissue and four tissues.

Additional file 6: Secondary structures of the putative miRNA precursors. DOCX document for the predicted secondary structures of the putative miRNA precursors.

Additional file 7: miRNA potential target genes. DOCX document for the predicted miRNA target genes.

Additional file 8: Occurrence of SSRs in P. ginseng unigenes. DOCX document for the summary of occurrence of SSRs in $P$. ginseng unigenes.

Additional file 9: Primer pairs for each SSR. XLSX document for the primer pairs for each SSR.

\section{Abbreviations}

P. ginseng: Panax ginseng C. A. Meyer; NGS: Next-generation sequencing; BLAST: Basic local alignment search tool; Nt: Nucleotide; Bp: Base pair; CDNA: Complementary DNA; AACT: Acetyl-CoA acetyltransferase; HMGS: HMG-CoA synthase; HMGR: HMG-CoA reductase; MVK: Mevalonate kinase; PMK: Phosphomevalonate kinase; MVD: Mevalonate diphosphate decarboxylase; GPS: Geranylgeranyl pyrophosphate synthase; IDI: Isopentenyl diphosphate isomerase; FPS: Farnesyl diphosphate synthase; SS: Squalene synthase; SE: Squalene epoxidase; AS: Amyrin synthase; DS: Dammarendiol synthase; UGT: UDP glycosyltransferase; CYP450: Cytochrome P450; EST: Expressed sequence tag; GO: Gene ontology; Nt: NCBI non-redundant nucleotide; Nr: NCBI non-redundant protein; KEGG: Kyoto encyclopedia of genes and genomes; NCBI: National center for biotechnology information; MFE: Minimal folding free energy; MFEl: Minimal folding free energy index miRNA: microRNA; pre-miRNA: miRNA precursor; AP2: APETALA2.

\section{Competing interests}

The authors declare that they have no competing interests.

\section{Authors' contributions}

CFL conceived the study, built the cDNA libraries, performed 454 sequencing, participated in the data analysis and drafted the manuscript. YJZ performed most of the data analysis. XG participated in 454 sequencing. CS contributed to designing the study. HML and JYS helped to conceive the study. YL participated in the data analysis. LZW participated in CDNA library construction and 454 sequencing. JQ performed the SSR analysis. SLC initiated the project, designed the study and participated in study coordination. All authors read and approved the final manuscript.

\section{Acknowledgements}

The authors thank Xiang Luo and Yunyun Niu for their assistance with sample collection. This work was supported by grants from the Jilin Province Key Science and Technology Project, the National Key Technology Research and Development Program of the Ministry of Science and Technology of China (No. 2011BAI03B01) and the Program for Changjiang Scholars and Innovative Research Teams in Universities of the Ministry of Education of China (No. IRT1150).

Received: 2 September 2012 Accepted: 2 April 2013

Published: 11 April 2013

\section{References}

1. Wee JJ, Mee PK, Chung AS: Biological activities of ginseng and its application to human health. In Herbal Medicine: Biomolecular and Clinical Aspects. 2nd edition. Edited by Benzie IFF, Wachtel GS. Boca Raton (FL): CRC Press; 2011. Chapter 8.

2. Chen CF, Chiou WF, Zhang JT: Comparison of the pharmacological effects of Panax ginseng and Panax quinquefolium. Acta Pharmacol Sin 2008 29:1103-1108.

3. Chen $S L$, Sun $Y Z$, Xu J, Luo HM, Sun C, He L, Cheng XL, Zhang BL, Xiao PG: Strategies of the study on herb genome program. Acta Pharm Sin 2010, 45:807-812.

4. Hattori J, Ouellet T, Tinker NA: Wheat EST sequence assembly facilitates comparison of gene contents among plant species and discovery of novel genes. Genome 2005, 48:197-206.

5. Jung JD, Park HW, Hahn Y, Hur CG, In DS, Chung HJ, Liu JR, Choi DW: Discovery of genes for ginsenoside biosynthesis by analysis of ginseng expressed sequence tags. Plant Cell Rep 2003, 22:224-230.

6. Sathiyamoorthy $\mathrm{S}, \mathrm{In}$ JG, Gayathri S, Kim YJ, Yang DC: Generation and gene ontology based analysis of expressed sequence tags (EST) from a Panax ginseng C. A. Meyer roots. Mol Biol Rep 2010, 37:3465-3472.

7. Sathiyamoorthy S, In JG, Gayathri S, Kim YJ, Yang D: Gene ontology study of methyl jasmonate-treated and non-treated hairy roots of Panax ginseng to identify genes involved in secondary metabolic pathway. Genetika 2010, 46:932-939.

8. Kim MK, Lee BS, In JG, Sun H, Yoon JH, Yang DC: Comparative analysis of expressed sequence tags (ESTs) of ginseng leaf. Plant Cell Rep 2006, 25:599-606.

9. Morozova O, Hirst M, Marra MA: Applications of new sequencing technologies for transcriptome analysis. Annu Rev Genomics Hum Genet 2009, 10:135-151.

10. Simon SA, Zhai J, Nandety RS, McCormick KP, Zeng J, Mejia D, Meyers BC: Short-read sequencing technologies for transcriptional analyses. Annu Rev Plant Biol 2009, 60:305-333.

11. Chen S, Luo H, Li Y, Sun Y, Wu Q, Niu Y, Song J, Lv A, Zhu Y, Sun C, Steinmetz A, Qian Z: 454 EST analysis detects genes putatively involved in ginsenoside biosynthesis in Panax ginseng. Plant Cell Rep 2011, 30:1593-1601 
12. Colaiacovo M, Subacchi A, Bagnaresi P, Lamontanara A, Cattivelli L, Faccioli P: A computational-based update on microRNAs and their targets in barley (Hordeum vulgare L.). BMC Genomics 2010, 11:595.

13. Jones-Rhoades MW, Bartel DP: Computational identification of plant microRNAs and their targets, including a stress-induced miRNA. Mol Cell 2004, 14:787-799.

14. Zhang $B$, Wang $Q$, Pan X: MicroRNAs and their regulatory roles in animals and plants. J Cell Physiol 2007, 210:279-289.

15. Zhang B, Wang Q, Wang K, Pan X, Liu F, Guo T, Cobb GP, Anderson TA: Identification of cotton microRNAs and their targets. Gene 2007, 397:26-37.

16. Zhang B, Pan X, Anderson TA: Identification of 188 conserved maize microRNAs and their targets. FEBS Lett 2006, 580:3753-3762.

17. Xie FL, Huang SQ, Guo K, Xiang AL, Zhu YY, Nie L, Yang ZM: Computational identification of novel microRNAs and targets in Brassica napus. FEBS Lett 2007, 581:1464-1474.

18. Bozhko M, Riegel R, Schubert R, Muller-Starck G: A cyclophilin gene marker confirming geographical differentiation of Norway spruce populations and indicating viability response on excess soil-born salinity. Mol Ecol 2003, 12:3147-3155.

19. Kim J, Jo BH, Lee KL, Yoon ES, Ryu GH, Chung KW: Identification of new microsatellite markers in Panax ginseng. Mol Cells 2007, 24:60-68.

20. Han JY, Kim HJ, Kwon YS, Choi YE: The Cyt P450 enzyme CYP716A47 catalyzes the formation of protopanaxadiol from dammarenediol-II during ginsenoside biosynthesis in Panax ginseng. Plant Cell Physiol 2011, 52:2062-2073

21. Sun C, Li Y, Wu Q, Luo H, Sun Y, Song J, Lui EM, Chen S: De novo sequencing and analysis of the American ginseng root transcriptome using a GS FLX Titanium platform to discover putative genes involved in ginsenoside biosynthesis. BMC Genomics 2010, 11:262.

22. Blake JA, Dolan M, Drabkin H, Hill DP, Ni L, Sitnikov D, Burgess S, Buza T, Gresham C, McCarthy F, Pillai L, Wang H, Carbon S, Lewis SE, Mungall CJ, Gaudet P, Chisholm RL, Fey P, Kibbe WA, Basu S, Siegele DA, Mclntosh BK, Renfro DP, Zweifel AE, Hu JC, Brown NH, Tweedie S, Alam-Faruque $Y$, Apweiler R, Auchinchloss A, et al: The Gene Ontology: enhancements for 2011. Nucleic Acids Res 2012, 40:D559-D564.

23. Gill SS, Tuteja N: Reactive oxygen species and antioxidant machinery in abiotic stress tolerance in crop plants. Plant Physiol Biochem 2010, 48:909-930.

24. Balachandran S, Yu X, Schobert C, Thompson GA, Lucas WJ: Phloem sap proteins from Cucurbita maxima and Ricinus communis have the capacity to traffic cell to cell through plasmodesmata. Proc Natl Acad Sci USA 1997, 94:14150-14155.

25. Alsheikh MK, Heyen BJ, Randall SK: Ion binding properties of the dehydrin ERD14 are dependent upon phosphorylation. J Biol Chem 2003, 278:40882-40889.

26. Lundqvist T, Schneider G: Crystal structure of activated ribulose-1,5bisphosphate carboxylase complexed with its substrate, ribulose-1,5bisphosphate. J Biol Chem 1991, 266:12604-12611.

27. Jasencakova Z, Meister A, Walter J, Turner BM, Schubert I: Histone H4 acetylation of euchromatin and heterochromatin is cell cycle dependent and correlated with replication rather than with transcription. Plant Cell 2000, 12:2087-2100.

28. Ma N, Xue J, Li Y, Liu X, Dai F, Jia W, Luo Y, Gao J: Rh-PIP2;1, a rose aquaporin gene, is involved in ethylene-regulated petal expansion. Plant Physiol 2008, 148:894-907.

29. Bartel DP: MicroRNAs: genomics, biogenesis, mechanism, and function. Cell 2004, 116:281-297.

30. Kozomara A, Griffiths-Jones S: miRBase: integrating microRNA annotation and deep-sequencing data. Nucleic Acids Res 2011, 39:D152-D157.

31. Xie F, Frazier TP, Zhang B: Identification and characterization of microRNAs and their targets in the bioenergy plant switchgrass (Panicum virgatum). Planta 2010, 232:417-434.

32. Zhang BH, Pan XP, Cox SB, Cobb GP, Anderson TA: Evidence that miRNAs are different from other RNAs. Cell Mol Life Sci 2006, 63:246-254.

33. Zhou M, Gu LF, Li PC, Song XW, Wei LY, Chen ZY, Cao XF: Degradome sequencing reveals endogenous small RNA targets in rice (Oryza sativa L. ssp. indica). Front Biol 2010, 5:67-90.

34. Frazier TP, Xie F, Freistaedter A, Burklew CE, Zhang B: Identification and characterization of microRNAs and their target genes in tobacco (Nicotiana tabacum). Planta 2010, 232:1289-1308.
35. Unver T, Parmaksiz I, Dundar E: Identification of conserved micro-RNAs and their target transcripts in opium poppy (Papaver somniferum L.). Plant Cell Rep 2010, 29:757-769.

36. Wang J, Yang $X, X u H, C h i X$, Zhang M, Hou X: Identification and characterization of microRNAs and their target genes in Brassica oleracea. Gene 2012, 505:300-308.

37. Aukerman MJ, Sakai $\mathrm{H}$ : Regulation of flowering time and floral organ identity by a MicroRNA and its APETALA2-like target genes. Plant Cell 2003, 15:2730-2741.

38. Chen Y, Yang X, He K, Liu M, Li J, Gao Z, Lin Z, Zhang Y, Wang X, Qiu X, Shen Y, Zhang L, Deng X, Luo J, Deng XW, Chen Z, Gu H, Qu LJ: The MYB transcription factor superfamily of Arabidopsis: expression analysis and phylogenetic comparison with the rice MYB family. Plant Mol Biol 2006, 60:107-124

39. Davletova S, Schlauch K, Coutu J, Mittler R: The zinc-finger protein Zat12 plays a central role in reactive oxygen and abiotic stress signaling in Arabidopsis. Plant Physiol 2005, 139:847-856.

40. Kantartzi SK, Ulloa M, Sacks E, Stewart JM: Assessing genetic diversity in Gossypium arboreum L. cultivars using genomic and EST-derived microsatellites. Genetica 2009, 136:141-147.

41. Park YH, Alabady MS, Ulloa M, Sickler B, Wilkins TA, Yu J, Stelly DM, Kohel RJ, El-Shihy OM, Cantrell RG: Genetic mapping of new cotton fiber loci using EST-derived microsatellites in an interspecific recombinant inbred line cotton population. Mol Genet Genomics 2005, 274:428-441.

42. SwissProt Database. http://www.uniprot.org/downloads.

43. InterPro Database. http://www.ebi.ac.uk/interpro/.

44. KEGG Database. http://www.genome.jp/kegg/.

45. Nr Database. ftp://ftp.ncbi.nih.gov/blast/db/FASTA/nr.gz.

46. Nt Database. ftp://ftp.ncbi.nih.gov/blast/db/FASTA/nt.gz

47. Thiel T, Michalek W, Varshney RK, Graner A: Exploiting EST databases for the development and characterization of gene-derived SSR-markers in barley (Hordeum vulgare L.). Theor Appl Genet 2003, 106:411-422.

48. Zeng S, Xiao G, Guo J, Fei Z, Xu Y, Roe BA, Wang Y: Development of a EST dataset and characterization of EST-SSRs in a traditional Chinese medicinal plant, Epimedium sagittatum (Sieb. Et Zucc.) Maxim. BMC Genomics 2010, 11:94.

49. Rozen S, Skaletsky H: Primer3 on the WWW for general users and for biologist programmers. Methods Mol Biol 2000, 132:365-386.

doi:10.1186/1471-2164-14-245

Cite this article as: Li et al:: Transcriptome analysis reveals ginsenosides biosynthetic genes, microRNAs and simple sequence repeats in Panax ginseng C. A. Meyer. BMC Genomics 2013 14:245.

\section{Submit your next manuscript to BioMed Central and take full advantage of:}

- Convenient online submission

- Thorough peer review

- No space constraints or color figure charges

- Immediate publication on acceptance

- Inclusion in PubMed, CAS, Scopus and Google Scholar

- Research which is freely available for redistribution 